\title{
High Throughput, High Quality Analysis in the Electron Microscope.
}

\author{
A. Hyde ${ }^{1}$, J. Goulden $^{1}$, N. Rowlands ${ }^{2}$, H.S.Ubhi ${ }^{1}$ \\ ${ }^{1}$ Oxford Instruments NanoAnalysis, Halifax Road, High Wycombe, HP12 3SE, UK \\ ${ }^{2}$ Oxford Instruments NanoAnalysis, 300 Baker Avenue, Concord, Massachusetts, USA
}

The rapid increase in detector throughputs and resolutions in recent years has been driven by user requirements for improved productivity without compromising the accuracy or quality of results.

One area where software development has improved productivity and data interpretation has been in the area of specimen characterization. This can often be time consuming and require highly skilled operators to interrogate the data. Oxford Instruments' AutoPhaseMap automatically finds areas of different characteristic composition from X-ray map data, and determines the distribution, area, constituent elements and composition of each of these areas or phases. In Fig.1 AutoPhaseMap has been used to characterize an aluminium alloy and its inter-metallic phases. For each phase identified by the software a phase image showing the distribution of the phase, a spectrum calculated by summing the pixels identified as belonging to that phase, and a quantitative result for each phase giving the average phase composition, is displayed. The AutoPhaseMap data is calculated in seconds, even for a large dataset with over 700,000 pixels, as used above. This allows AutoPhaseMap to be used in real-time as part of the specimen investigation, in addition to being used to analyze the phases in the sample.

EBSD requirements are continually expanding as the technique becomes more widespread. Faster data acquisition speeds are increasingly important in reducing the time spent collecting data on the SEM. EBSPs can be collected and solved at many 100's of patterns per second, even at lower beam energies. The Nordlys $\mathrm{Max}^{2}$ detector acquires and solves EBSD patterns in real time at up to 870 patterns per second, simultaneously with EDS data. This speed of acquisition is critical in many applications areas; for example in collecting statistically representative data sets over large areas, as is required for determining macro texture. Figure 2 shows an inverse pole figure map collected from a zirconium tube in which over 16000 grains were measured.

EDS and EBSD technology is being improved to allow ever larger datasets collected from larger areas or at higher speeds to be analysed more efficiently, while maintaining high quality results. Examples of these changes can be seen at all sample scales from larger than the minimum magnification on a WSEM right the way through to aberration corrected TEM. The latest hardware and software developments from Oxford Instruments will be placed in the context of this requirement for efficiency and accuracy in this presentation. 
Fig 1
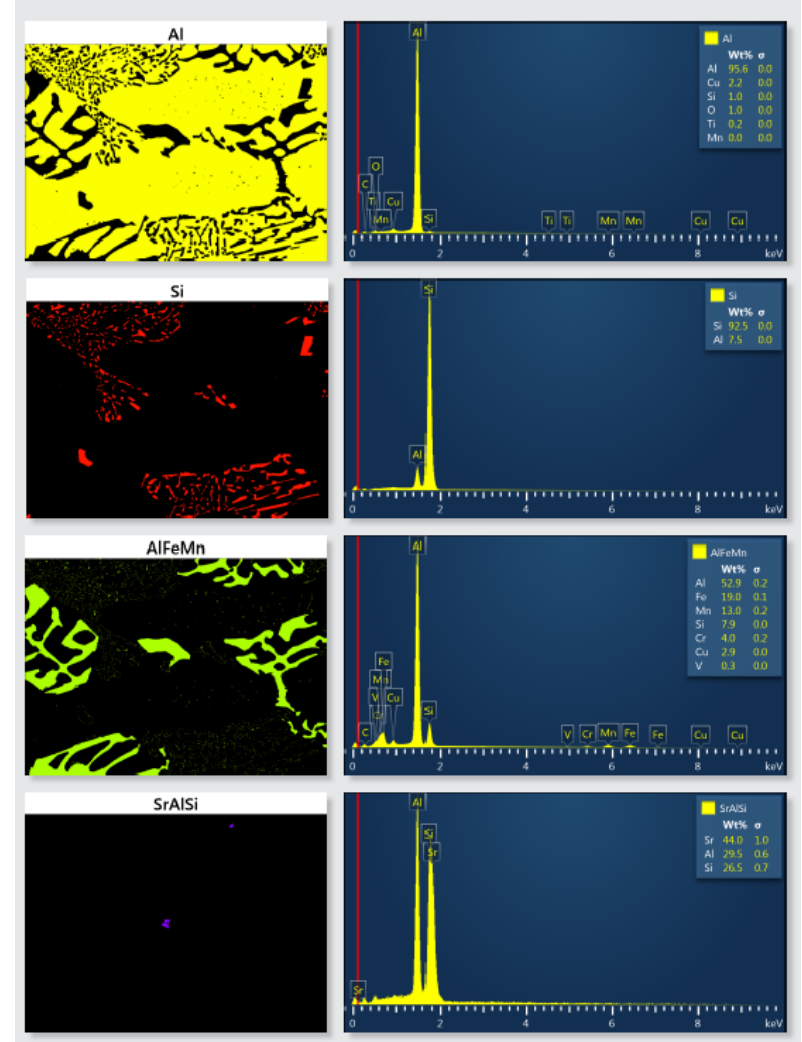

Fig. 4. Autophaselapp results for the four phases identifed in the aluminium alloy sample

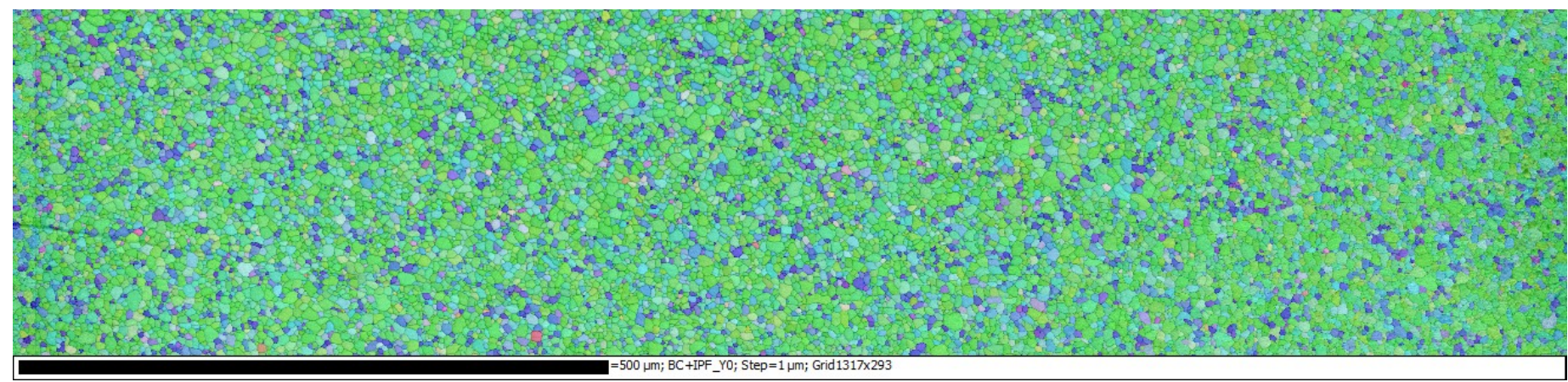

Figure 2 Inverse Pole Figure Map, showing the strong texture in a zirconium tube. 